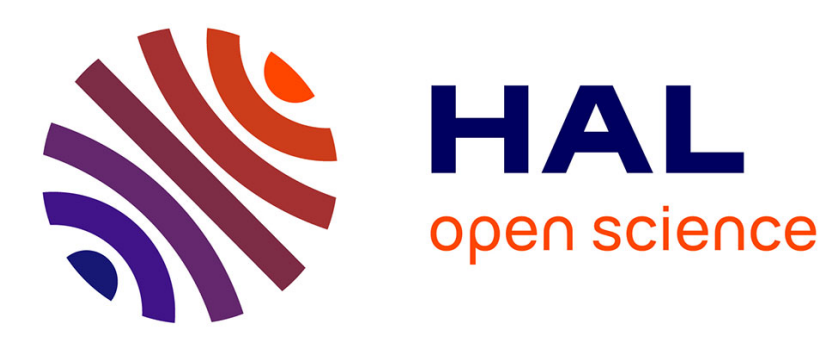

\title{
Intelligent control for a drone by self-tunable fuzzy inference system
}

\author{
Kadda Zemalache, Hichem Maaref
}

\section{To cite this version:}

Kadda Zemalache, Hichem Maaref. Intelligent control for a drone by self-tunable fuzzy inference system. 6th International Multi-Conference on Systems, Signals and Devices (SSD 2009), Mar 2009, Djerba, Tunisia. (elec. proc.), 10.1109/SSD.2009.4956805 . hal-00965125

\section{HAL Id: hal-00965125 \\ https://hal.science/hal-00965125}

Submitted on 24 Mar 2014

HAL is a multi-disciplinary open access archive for the deposit and dissemination of scientific research documents, whether they are published or not. The documents may come from teaching and research institutions in France or abroad, or from public or private research centers.
L'archive ouverte pluridisciplinaire HAL, est destinée au dépôt et à la diffusion de documents scientifiques de niveau recherche, publiés ou non, émanant des établissements d'enseignement et de recherche français ou étrangers, des laboratoires publics ou privés. 


\title{
INTELLIGENT CONTROL FOR A DRONE BY SELF-TUNABLE FUZZY INFERENCE SYSTEM
}

\author{
K. M. Zemalache ${ }^{1}$ and H. Maaref ${ }^{2}$ \\ ${ }^{1}$ Université des Sciences et de la Technologie d'Oran M B \\ B.P. 1505 Oran El M'Naouer, Algérie \\ e-mail: zemalache@hotmail.com \\ ${ }^{2}$ Laboratoire d'IBISC, CNRS-FRE 3190 \\ Université d'Evry Val d'Essonne \\ 40, rue du Pelvoux, 91020, Evry Cedex, France \\ e-mail: maaref@iup.univ-evry.fr
}

\begin{abstract}
The work describes an automatically on-line Self-Tunable Fuzzy Inference System (STFIS) of a new configuration of mini-flying called XSF (X4 Stationnary Flyer) drone. A Fuzzy controller based on on-line optimization of a zero order Takagi-Sugeno fuzzy inference system (FIS) by a back propagation-like algorithm is successfully applied. It is used to minimize a cost function that is made up of a quadratic error term and a weight decay term that prevents an excessive growth of parameters. Thus, we carried out control for the continuation of simple trajectories such as the follow-up of straight lines, and complex (half circle, corner) by using the STFIS technique. This permits to prove the effectiveness of the proposed control law. We studied the robustness of the two controllers used in the presence of disturbances. We presented two types of disturbances, the case of a gust of wind and taking into account white noise disturbances. A comparison between the Self-Tunable Fuzzy Inference System (STFIS) and Adaptive Network based Fuzzy Inference System (ANFIS) is given.
\end{abstract}

Index Terms - Self-Tunable Fuzzy Inference System, Static Feedback Linearization controller, Tracking control, Drone.

\section{INTRODUCTION}

The past few years have witnessed a rapid growth in the use of fuzzy logic controllers for the control of processes, which are complex and badly defined. Most fuzzy controllers developed till now have been of the rule-based type, where the rules in the controller attempt to model the operator's response to particular process situation.

Recently, the resurgence of interest in the field of artificial neural networks has injected a new driving force into the fuzzy literature. The back-propagation learning rule, which drew little attention till its applications to artificial neural networks was discovered, is actually an universal learning paradigm for any smooth parameterized models, including fuzzy inference systems. As a result, a fuzzy inference system can now not only take linguistic information from human experts, but also adapt itself using numerical data (input/output pairs) to achieve better performance. This gives fuzzy inference systems an edge over neural networks, which cannot take linguistic information directly.

In autonomous wheeled robot, many developed learning techniques have arisen in order to generate or to tune fuzzy rules. Most of them are based on the so-called "NeuroFuzzy learning algorithms" as proposed by [10, 12, 13, 17]. These methods are well for constructing an optimal fuzzy system model which is used to identify the corresponding practical system.

The stabilization problem of a four rotors rotorcraft is also studied and tested by Castillo [5] where the nested saturation algorithm is used, the input/output linearization procedure [7], in [4] a proportional integral derivative (PID) controller and a linear quadratic (LQ) controller were implemented and proved capable of regulating the system. Hanford et al [8], presented a simple closed loop equipped with MEMS (Micro-Electro-Mechanical Systems) sensors and PIC based processing unit. Waslander et al [18] maked an emphasis on the insufficiency of classical control methods and proposes the integral sliding mode controller associated with reinforcement learning to achieve multi agent control. Tayebi and McGilvray [16] proposed a new quaternion based feedback control scheme for exponential attitude stabilization of a quadrotor. The proposed controller is based upon the compensation of the Coriolis and gyroscopic torques and the use of a $P D^{2}$ feedback structure, where the proportional action is in terms of the vector quaternion and the two derivative actions are in terms of the airframe angular velocity and the vector quaternion velocity. Hoffman et al [9], achieved the formation control by sliding mode technique and focused on collision and obstacle avoidance by extracting the state variables with a Kalman filter. Bestaoui et al [2] addressed the problem of 
characterizing maneuvers paths on the group of rigid body motions in $3 \mathrm{D}$ for a quadrotor. The role of the trajectory generator is to generate a feasible time trajectory for the UAV.

All the reviewed techniques require the well knowledge of the system dynamic model and parameters. In this paper, a STFIS control strategy is developed based on the systems output measures is implemented. This technique early used for autonomous wheeled robot, is adapted and modified for the used with the XSF.

The arrangement of this paper is as follows. The Presentation of the XSF drone is given in the second section. The developed ideas of control for the XSF by the Self-Tunable Fuzzy Inference System (STFIS) controller is presented in the third section. Motion planning and simulation results are introduced in the fourth section. The robustness of the proposed controller is then evaluated and compared with a Static Feedback Linearization controller (SFL) to stabilize the XSF by using the point to point steering stabilization in the fifth section. Finally, conclusion and future works are given in the last section.

\section{PRESENTATION OF THE DRONE XSF}

Modeling and controlling aerial vehicles (blimps, mini rotorcraft) are the principal preoccupation of the IBISC-group. Within this optic, who attracted the contest of the DGAONERA $^{1}$ was the XSF project which consists of a drone with revolving aerofoils, (see Fig.1 left). It is equipped with four rotors where two are directionals, what we call in the following X4 Stationary Flyer (XSF).

The XSF is an engine of $68 \mathrm{~cm} \times 68 \mathrm{~cm}$ of total size and
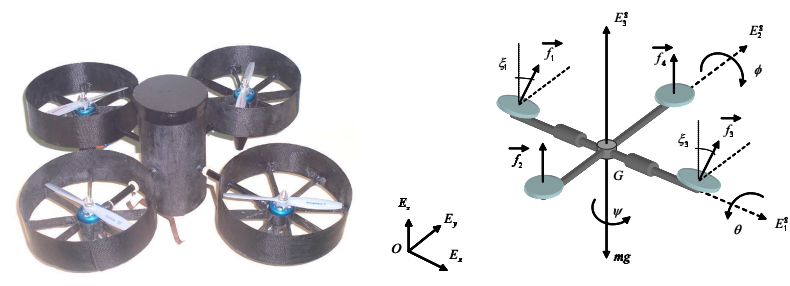

Figure 1. Conceptual form of the XSF (left), Frames attached to the XSF (right)

not exceed $2 \mathrm{~kg}$ in mass. It is designed in a cross form and made of carbon fiber. Each tip of the cross has a rotor including an electric brushless motor, a speed controller and a two-blade ducted propeller. The operating principle of the XSF can be presented thus: two rotors turn clockwise, and the two other rotors turn counterclockwise to maintain the total equilibrium in yaw motion. The equilibrium of angular velocities of all rotors done, the Unmanned Aerial Vehicle (UAV) is either in stationary position, or moving vertically. A characteristic of the XSF compared to the existing quadrotors, is the swiveling of the supports of the

\footnotetext{
${ }^{1}$ This work is supported by the mini-flyer competition program organized by the DGA (Direction Générale des Armements) and the ONERA (Office Nationale d'Etude et de Recherche en Aérospatiale), France.
}

motors 1 and 3 around the pitching axis thanks to two small servomotors controlling the swiveling angles $\xi_{1}$ and $\xi_{3}$. This swiveling ensures either the horizontal rectilinear motion or the rotational movement around the yaw axis or a combination of these two movements which gives the turn (see Fig. 1 right). This permits a more stabilized horizontal flight and a suitable cornering.

\section{SELF-TUNABLE FUZZY INFERENCE SYSTEM}

The aim in this section is to make comparison between model based approaches and experts analysis involving fuzzy systems. Classical model based techniques such us the Static Feedback Linearization techniques have been investigated and used for stabilization with motion planning [20].

The formal analogy between a fuzzy inference system and a multilayer neural network associated with optimization algorithms is used from the retro-propagation gradient algorithm have winded up in what is called a STFIS Network.

\subsubsection{Presentation of STFIS}

A Takagi-Sugeno type fuzzy system is determined in three stages [15]:

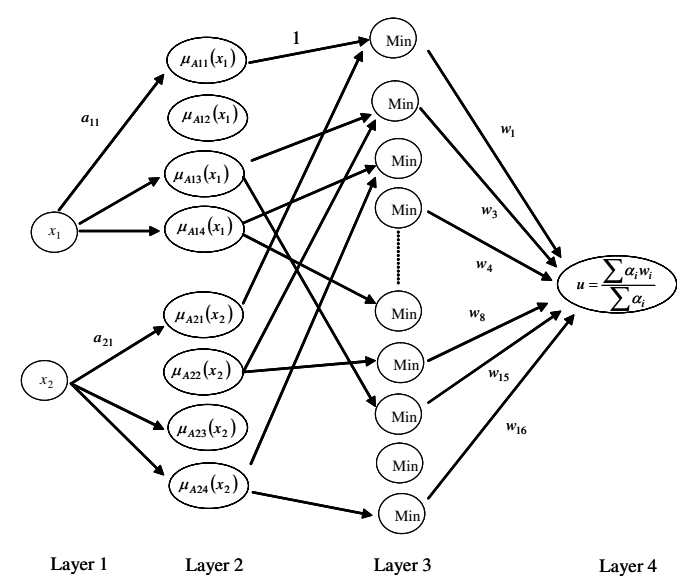

Figure 2. Self-Tunable Fuzzy Inference System

1. Given an input $x$ a membership degree $\mu$ is obtained from the antecedent part of rules.

2. A truth value degree $\alpha_{i}$ is obtained, associated to the premises of each rule $R_{i}$ : IF $x_{1}$ is $X_{1}$ AND IF $x_{2}$ is $X_{2}$ THEN $u$ IS $w_{i}$.

3. An aggregation stage to take in account all rules by $u=\sum_{i=1}^{r} \alpha_{i} w_{i} / \sum_{i=1}^{r} \alpha_{i}$ permit to obtain a crisp value $u$.

These operations can be traduced by the layer structure shown in Fig. 2. Each layer, connected with others by adjustable parameters, having a specific function. 


\subsubsection{Architecture and Learning Algorithm}

In this work, we propose to generate the fuzzy control rules by an optimization method, which is done entirely on-line. Jordan [11] proposes the distal control method, which is used by [14] under the name of JEAN (Jordan method Extended for Adaptive Neuro-control). This architecture (Fig. 3 left) needs the used of two STFIS networks:

1. a first networks to identify the drone (Model);

2. a second networks to control the drone (Controller).

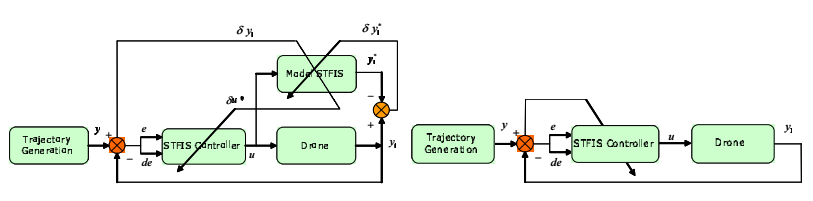

Figure 3. JEAN Learning Architecture (left), Control architecture mini-JEAN (right)

For the control of the XSF, we have used the architecture known as the "mini-JEAN" as illustrated in the Fig. 3 (right) [14]. This architecture not require an emulator network. It uses only one network as a controller, the learning of which is done directly by the back-propagation of the output error.

Compared to the architecture JEAN, some equivalent performances are obtained for the mean error in generalization. On the other hand, the computing time favors clearly mini-JEAN [14]. Optimization of adjustable parameter is accomplished with a version of the classic gradient retropropagation algorithm adapted to net structure of Fig. 3 (right).

The aim is to minimise cost function $E$ :

$$
E=\frac{1}{2} e^{2}
$$

where $e$ is the difference between the process output and the set point. The basic equations of the algorithm are:

$$
\begin{gathered}
w_{i j}^{n}(t)=w_{i j}^{n}(t-1)+\Delta w_{i j}^{n}(t) \\
\Delta w_{i j}^{n}(t)=-\eta \delta_{i}^{n} \alpha_{j}^{n-1}+b \Delta w_{i j}^{n}(t-1)
\end{gathered}
$$

Where :

$w_{i j}^{n}(t): i^{t h}$ parameter between $i$ of layer $n$ and $j^{s t}$ unit of layer n-1.

$\eta$ : learning gain.

$t$ : training iteration.

$b:$ moment parameter.

$\delta_{i}^{n}$ : error term ( $i^{t h}$ neurone of layer $\left.\mathrm{n}\right)$.

$\alpha_{j}^{n-1}$ : output of $j^{\text {th }}$ unit of layer n-1.

The quality of solution obtained using this algorithm depends on input learning signals, algorithm control parameters and learning duration (number of iterations).

\subsubsection{Algorithm Modification Weight Regression}

The procedure is entirely done on-line on the engine. The table of rules (weights $w_{i}$ ) can be initially empty or filled with an a priori knowledge. The engine acquires by its systems output measures, calculates the error to the backpropagated, updates the triggered rules on-line. The weights of the table of decision are then adjusted locally and progressively.

The cost function is given by:

$$
J=E+\lambda \sum w_{i}^{2}
$$

where $E$ is the classic quadratic error, $w$ are the parameters (weights) to optimize parameters and $\lambda$ is a constant that controls the growth of parameters. The second term in $J$ is known as weight decay and used usually in the context of classification problems. This technique has been analyzed in the framework of learning theory and it was shown that is a very simple manner to implement a regularization method in a neural network in order to optimize the compromise between the learning error and the generalization error $[3,6]$. Thanks to the classic back-propagation algorithm, the parameters are modify as:

$$
w(t+1)=w(t)+\eta\left(\frac{-\partial J}{\partial w}\right)
$$

This algorithm easily includes the effect of the second term of the cost function $J$ and by taking $\beta=2 \lambda \eta$ (regression coefficient) we obtain:

$$
w(t+1)=w(t)+\eta\left(\frac{-\partial E}{\partial w}\right)-\beta w(t)
$$

Since a fuzzy inference system is concerned, we adapt this formula by multiplying $\beta$ by the firing term of the rule, namely $\alpha_{i} / \sum \alpha_{i} . \alpha_{i}$ is the truth value of the premise part of the triggered rule.

If we limit the optimization only on the conclusions parameters $w_{1 j}^{4}$. Then, we get

$$
\begin{aligned}
& \Delta w_{1 j}^{4}(t)=-\eta \delta_{1}^{4} \alpha_{j}^{3}+b \Delta w_{1 j}^{4}(t-1) \\
& -\alpha_{j}^{3} 2 \eta \lambda w_{1 j}^{4}(t-1) / \sum_{j} \alpha_{j}^{3}
\end{aligned}
$$

with

$$
\delta_{1}^{4}=y-y_{1} / \sum_{j} \alpha_{j}^{3}
$$

Where:

$y_{1}$ :effective output value.

$y$ :desired output.

\section{MOTION PLANNING AND SIMULATION RESULTS}

The XSF is tested in simulation in order to validate some motion planning algorithm considering the proposed STFIS control laws. We have considered a total mass equal to $m=2 \mathrm{~kg}$. The technical characteristics of this flying vehicle were presented in [?]. We solve the tracking control problem using the point to point steering stabilization see $[1,19]$ for more details.

A Fuzzy controller based on an on-line optimization of a 
zero order Takagi-Sugeno fuzzy inference system is successfully applied. It is used to minimize a cost function that is made up of a quadratic error term and a weight decay term that prevents an excessive growth of parameters of the consequent part. The main idea is to generate the conclusion parts (so-called weight) of the rules automatically thanks to an optimization technique. The used method is based on a back-propagation algorithm where the parameters values are free to vary during the optimization process.

Starting with a preinitialized rules table, when XSF begins to fly, it performs the acquisition of the distances (observations), calculates the cost function to back-propagation, updates the triggered rules in real time, begins to move and so on. The weights $w_{i}$ are then adjusted locally and progressively. The shape of the used membership functions is triangular and fixed in order to extract and represent the knowledge from the final results easily. The deduce the truth value, we use the MIN operator for the composition of the input variables. For the control of the XSF, we use the architecture known as "mini-JEAN". The universes of discourse are normalized and shared in five fuzzy subsets for all displacement.

The linguistic labels are defined as follows: NB: Negative Big, NS: Negative Small, Z: approximately Zero, PS: Positive Small and PB: Positive Big.

The results of the simulation are reported in the table 1 for $z$ displacement. The optimization phase tends to stable

Table 1. Weight for $z$ displacement

\begin{tabular}{|c|c|c|c|c|c|}
\hline de $\backslash \mathrm{e}$ & NB & NS & Z & PS & PB \\
\hline PB & 29.62 & 29.71 & 7.59 & 2.55 & 2.99 \\
\hline PS & 29.52 & 31.34 & 10.63 & 3.02 & 1.69 \\
\hline Z & 34.22 & 29.73 & 19.54 & 4.84 & 2.79 \\
\hline NS & 39.72 & 41.37 & 22.32 & 1.74 & 9.61 \\
\hline NB & 39.92 & 39.82 & 28.27 & 7.96 & 9.62 \\
\hline
\end{tabular}

Table 2. Learning linguistic table

\begin{tabular}{|c|c|c|c|c|c|}
\hline de $\backslash \mathrm{e}$ & NB & NS & Z & PS & PB \\
\hline PB & B & B & W & VW & VW \\
\hline PS & B & B & W & VW & VW \\
\hline Z & VB & B & M & VW $^{*}$ & VW \\
\hline NS & VB & VB & M $^{*}$ & VW $^{*}$ & W \\
\hline NB & VB & VB & B & W & W \\
\hline
\end{tabular}

weights (Fig. 4 left). In these circumstances the outputs linguistic labels could be interpreteted as follows (Fig. 4 right):

VW: [1 , 5] Very Weak, W: [7, 11] Weak, M: [19, 23] Medium, B: [28, 32] Big and VB: [34 , 42] Very Big.

The table 2, illustrates the linguistic translation of the table obtained by on-line optimization for the $z$ displacement (table 1). By comparing the table proposed by learning and by human expertise (see table 2 and table 3 ), we can
Table 3. Expertise linguistic table

\begin{tabular}{|c|c|c|c|c|c|}
\hline de $\backslash \mathrm{e}$ & NB & NS & Z & PS & PB \\
\hline PB & B & B & W & VW & VW \\
\hline PS & B & B & W & VW & VW \\
\hline Z & VB & B & M & W & VW \\
\hline NS & VB & VB & B & W & W \\
\hline NB & VB & VB & B & W & W \\
\hline
\end{tabular}

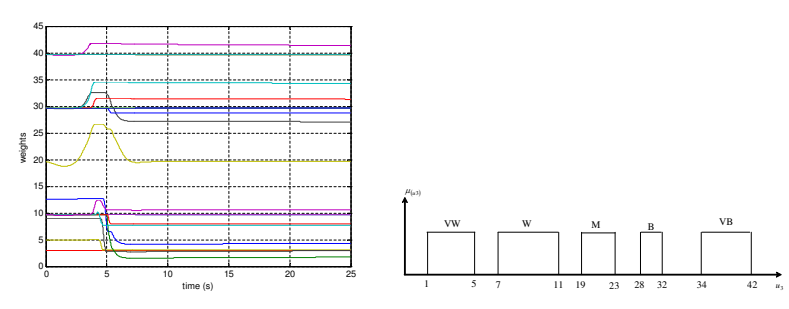

Figure 4. Evolution of the weights for $z$ displacement (left), Representation of the linguistic translation of the controller $u_{3}$ (right).

observe that the two sets of linguistic rules are quite close. Three cases (noted with *) are different and they differ from only one linguistic concept (M instead B and VW instead W). So, we can claim that the extracted rules are quite logical and coherent. On the other hand, the main advantage of the described technique is the optimization of the controller with respect to the actual characteristics of the engine.

The use of a function cost gathering a quadratic error and a term of regression of the weights enabled us to achieve our goal. For this behavior, the building of the navigation controller is done entirely on-line by the optimization of a zero order Takagi-Sugeno fuzzy inference system (FIS) by a back-propagation-like algorithm.

Fig.5 (right) illustrates the controlled positions $z x y$ using STFIS controller where $u_{3}$ and $u_{2}$, denote the command signals for $z, x$ or $y$ directions respectively. Note that the input $u_{3}=m g$ at the equilibrium state is always verified. The inputs $u_{2}$ tend to zero after having carried out the desired orientation of the vehicle. It is also shown in this figure that we can stabilize the system to make a following movement by the swivelling of the engine actuators 1 and 3 .

The 3D displacement is depicted with straight, arc, round corners like connection and realization of a helical trajectory (see Fig.5 (left) and Fig. 6).

As comparison, we used also the ANFIS algorithm. According to the found results, Fig. 7 (left), we can say that this approach ensures the vertical flight of the drone with a very weak static error but any time it does not preserve the legibility of the rules. In addition, controller STFIS (Fig. 7 (right)) is compared with method ANFIS. The drone is controlled to accomplish vertical flight according to the direction $z$ by using both technique. Controller STFIS has an advantage, which is the legibility of 

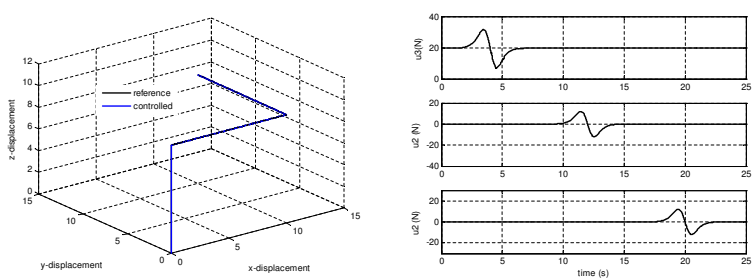

Figure 5. Realization of a straight corners (left), Inputs $u_{3}$ and $u_{2}$ for the realization of a straight corners (right)
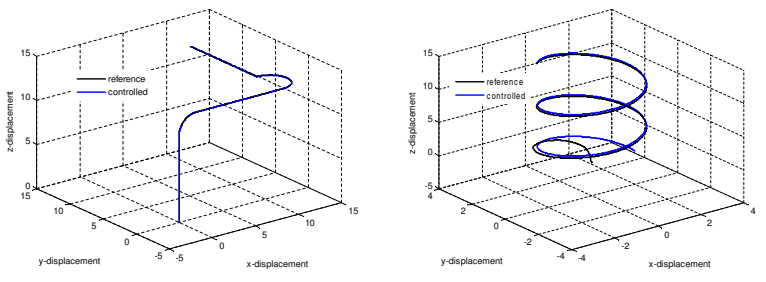

Figure 6. Realization of a round corners (left) and helical trajectory (right).

the rules (tables 1 and 2) contrary to the controller ANFIS who is a black box.
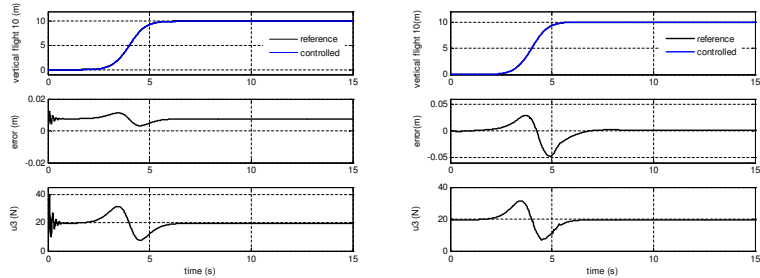

Figure 7. Realization of a $z$ displacement by ANFIS controller (left) and STFIS (right)

\section{CONTROLLERS ROBUSTNESS}

\subsection{Disturbances with wind influence}

The robustness of the developed controllers are evaluated regarding external disturbances and performance degradations in the case of wind influence. In the case of the XSF, a resistance or a drag force is opposed to its movement in flight. The work produced by this force involves an additional energy consumption at the actuators levels which limits its maneuvering capacities in flight. This force can be expressed as follow:

$$
F_{i}=\frac{1}{2} C_{x} \rho A V_{i}^{2}
$$

where $F_{i}[\mathrm{~N}]$ is the drag force following the $i$ axis, $V_{i}$ $[\mathrm{m} / \mathrm{s}]$ is the drone velocity, $A\left[\mathrm{~m}^{2}\right]$ is the coss-sectional area perpendicular to the force flow and $\rho\left[\mathrm{Kg} / \mathrm{m}^{3}\right]$ is the body density. The equation 9 induced a drag coefficient $C_{x}$ which is a dimensionless quantity that describes a characteristic amount of aerodynamic drag depending on the XSF structure and which is experimently determined by windtunnel tests. This coefficient is equal to 0.5 for the $x$ and $y$ directions and 0.08 for the $z$ displacement. The surface characteristic $A$ of the XSF drone is equal to $A=0.031 \mathrm{~m}^{2}$ and it density is considered equal to $\rho=1.22 \mathrm{Kg} / \mathrm{m}^{3}$. The Fig. 8 present the simulation re-
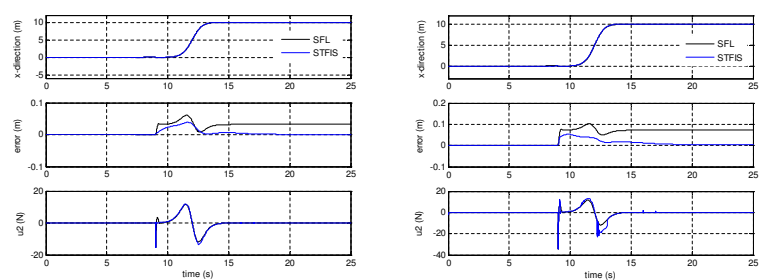

Figure 8. Wind influence with a drag force of $1.4 N$ (left) and $2.1 N$ (right) for the $x$ direction.

sults in the case of a drag force of $F_{d g}=1.4 \mathrm{~N}$ and of $F_{d g}=2.1 N$ according to the $x$ displacement. The STFIS controller exhibits chattering signal problems in the transition phase while the SFL controller presents static errors that varies proportionally with the drag force amplitude $F_{d g}$. The same observations are found according to the two directions $y$ and $z$.

\subsection{White noise disturbances}

The robustness study was realized in simulations taking into account disturbances with a white noise. We considered two cases, in the first one, the noise power is equal to 0.5 and 2 decibel in the second case for both SFL and STFIS controller along the $z$ direction. To see the behavior of the two controllers according to noise measurement, the Fig.9, shows the vertical flight allure for both controllers. It is noticed that the STFIS controller gives good results compared to the SFL controller.
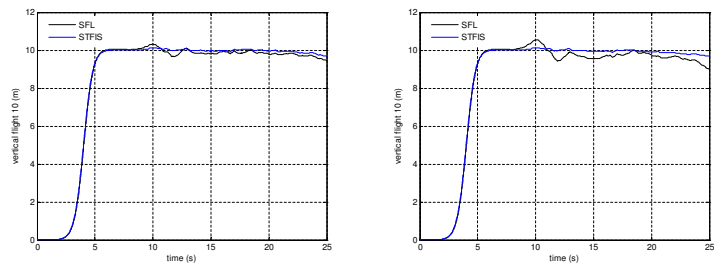

Figure 9. Vertical flight with noise power $0.5 \mathrm{~dB}$ (left) and $2 \mathrm{~dB}$ (right) for both controllers.

\section{CONCLUSION}

In this paper, we studied a new configuration of flyer engine called XSF. We have considered in this work the stabilizing/tracking control problem for the three decoupled 
displacements of a XSF. The objectives are to test the capability of the engine to fly with straight, arc, rounded intersections and complex trajectories (helical).

We have presented and implemented an optimization technique allowing an on-line adjustment of the fuzzy controller parameters. The descent gradient algorithm, with its capacities to adapt to unknown situations by the means of its faculties of optimization, and the fuzzy logic, with its capacities of empirical knowledge modelling, are combined to control a new configuration of flyer engine. Indeed, we have obtained an on-line optimized Takagi-Sugeno type FIS of zero order. This method is simple, economical and safe since it is done on a mini-flying robots. It leads to very quick and efficient optimization technique. A comparison between the STFIS set rules and that deduced by human expertise, shows the validity of the proposed technique.

An analysis of the STFIS (which not require the good knowledge of the model) and the SFL (requires the well knowledge of the system model and parameters) controllers and their robustness regarding disturbances, shows the advantages and the disadvantages of these two techniques.

\section{REFERENCES}

[1] L. Beji, A. Abichou and K. M. Zemalache, "Smooth control of an X4 bidirectional rotors flying robots," Fifth International Workshop on Robot Motion and Control, Dymaczewo, Poland, June 2005.

[2] Y. Bestaoui and R. Slim, "Maneuvers for a QuadRotor Autonomous Helicopter," AIAA, Rohnert Park, California, 7-10 May 2007.

[3] C. M. Bishop, "Regularization and complexity control in feedforward neural networks," Proc. IEEE Int. Conf. on Neural Networks, Paris, Vol. 1, PP. 141-148, 1995.

[4] S. Bouabdallah, A. Noth and R. Siegwart, "PID vs LQ control techniques applied to an indoor micro quadrotor," Proceeding of the IEEE, International Conference on Robotics and Automation, Barcelona, Spain, PP 2259-2264, April 2005.

[5] P. Castillo, A. Dzul and R. Lozano, "Real-time stabilization and tracking of a four rotor mini-rotorcraft," IEEE Transactions on Control Systems Technology, Vol. 12, N 4, pp 510-516, July 2004.

[6] M.Y. Chow, "An analysis of weight decay as a methodology of reducing three-layer feedforward artificial neural network for classification problems," ICNN'94, Proc. IEEE Int. Conf. on Neural Networks, Orlando, Vol. 1, PP. 600-605, 1995.

[7] J. Hauser, S. Sastry and G. Meyer, "Nonlinear control design for slightly non-minimum phase systems: application to V/STOL aircraft," Automatica, 28, 04, pp 665-679 1992.
[8] S.D. Hanford, L.N. Long and J.F. Horn, "A Small Semi-Autonomous Rotary-Wing Unmanned Air Vehicle (UAV)," AIAA2005-7077.

[9] G. Hoffmann, D. G. Rajnarayan, S. L. Waslander, D. Dostal, J. C. Jang and C. J. Tomlin, "The stanford testbed of autonomous rotorcraft for multi-agent control (STARMAC)," 23rd Digital Avionics System Conference, Salt Lake City, UT, November 2004.

[10] J.S.R. Jang, "ANFIS: adaptive-network-based fuzzy inference system," IEEE Transactions System, Man and Cybernet.23, pp 665-685, 1993.

[11] M. I. Jordan and D. Rumelhart, "Internal world models and supervised learning," Prog. of the eighth Int. Workschop on Machine Learning, PP. 70-74 1991.

[12] H. Maaref and C. Barret, "Sensor-based navigation of a mobile robot in an indoor environment," Robotics and Autonomous Systems, 38, pp 1-18, 2002.

[13] H. Nomura, I. Hayashi and N. Wakami, "A selftuning method of fuzzy control by descent method," Proc. 4th IFSA Congr., Brussels, PP 155-158, 1991.

[14] J. M. Renders, "Mtaphore biologiques appliques la commande de processus," Thse de doctorat, Universit libre de Bruxellese, Bruxellese, 1994.

[15] T. Takagi and M. Sugeno, "Derivation of Fuzzy control rules from human operator's control actions," in Proc. IFAC Symp. On Fuzzy Information, Knowledge Representation and Decision Analysis, July, PP. 5560, 1983.

[16] A. Tayebi and S. McGilvray, "Attitude Stabilization of a VTOL Quadrotor Aircraft," IEEE Transactions on Control Systems Technology, Vol. 14, N 3, May 2006.

[17] L. X. Wang and J. M. Rendel, "Back-propagation fuzzy system as nonlinear dynamic system identifiers," Proc. IEEE, Int. Conf. on fuzzy system, San Diego, PP 1409-1416, 1991.

[18] S. L. Waslander, G. Hoffmann, J. S. Jang and C. J. Tomlin, "Multi-agent X4-flyer testbed control design: integral sliding mode vs. reinforcement learning," IEEE/RSJ International Conference on Intelligent Robots and Systems, PP 468-473 2005.

[19] K. M. Zemalache, L. Beji and H. Maaref, "Two Inertial Models of X4-Flyers Dynamics, Motion Planning and Control," Journal of the Integrated ComputerAided Engineering (ICAE), Vol. 14, N. 2, pp. 107119, January 2007.

[20] K. M. Zemalache, L. Beji and H. Maaref, "Control of a Drone: Study and Analysis of the Robustness," Journal of Automation, Mobile Robotics and Intelligent Systems, Vol.2, N 1, pp 33-42, January 2008. 